\title{
DT4GITM - A Vision for a Framework for Digital Twin enabled IT Governance
}

\author{
Geert Poels \\ Ghent University, \\ Ghent, Belgium \\ geert.poels@ugent.be
}

\author{
Henderik A. Proper \\ Luxembourg Institute of Science and Technology, \\ and University of Luxembourg \\ e.proper@acm.org
}

\author{
Dominik Bork \\ TU Wien, \\ Vienna, Austria \\ dominik.bork@tuwien.ac.at
}

\begin{abstract}
This paper is concerned with the question of how novel digital technologies can be used to enable IT governance to better deal with the need for more agility, flexibility, adaptivity, and connectivity, as brought about by our modern day society. We propose to digitally transform IT governance, in particular making it smart(er) by following a data-driven approach. In line with this, we present a vision for digitally transformed IT governance in the form of the DT4GITM (Digital Twin for Governed IT Management) framework, which exploits the Digital Twin concept as it is already used in other fields to monitor, analyze, simulate, and predict the performance of real-world assets. The purpose of the DT4GITM framework is to serve as a reference architecture for a technological infrastructure based on the Digital Twin concept that connects three interrelated systems - the IT governance processes, the governed IT management processes, and the managed organizational IT assets.
\end{abstract}

\section{Introduction}

The Digital Age puts extreme requirements regarding agility, flexibility, adaptivity, and connectivity, on the system of organizational IT assets and their associated management processes. This, in turn, poses challenges to the IT governance system in dealing with the resulting frequent changes, or rather, continuous evolution, of the IT (management) system. We conceptualize the IT governance system as the whole of interrelated organizational governance assets (i.e., organizational structures, processes, relational mechanisms [1]) that are purposefully combined to govern the combination of (1) the system of organizational IT assets, and (2) their management processes, to ensure strategic alignment, risk management, and compliance [2].

Despite proposals to introduce agility into the IT governance implementation process [3], little is known about how easy it is to adapt IT governance systems to deal with frequent changes in the system of organizational IT assets and/or their management processes [4]. IT governance frameworks have also been criticized for a lack of flexibility in their support of IT governance implementation [5, 6]. We, furthermore, observe there to be a lack of knowledge of how actual IT governance system implementations are supported by IT, apart from the proverbial use of spreadsheet applications.

The goal of this paper is to present and illustrate a vision for digital transformation of IT governance. Although our research is still in an early phase, we believe that this vision has the potential to make IT governance more apt to address the challenges that come with digital transformation and the corresponding changes to the organizational IT assets and their management processes. To this end, the paper presents the DT4GITM (Digital Twin for Governed IT Management) framework, which exploits the concept of Digital Twin [7] to make IT governance systems smarter by following a data-driven approach. The purpose of the DT4GITM framework is to provide a reference architecture for a technological infrastructure that applies the Digital Twin concept to connect three interrelated systems - the IT governance processes, the governed IT management processes, and the managed organizational IT assets - using bi-directional data streams that are implemented through sensors and actuators, and that offers monitoring and prediction capabilities for reactive and proactive modes of IT governance. This application is what we refer to in the paper briefly as an IT governance digital twin.

The proposed framework articulates our vision by defining the different components of an IT governance digital twin in terms of their purpose, function, and relationships with other components as well as the system's environment. The data-driven analytics provided by an IT governance digital twin is based on an integration of heterogeneous data, including data from external sources, and is made possible, in our vision, through the use of Knowledge Graphs [8] and related technology. To the best of our knowledge, the application of digital twins and knowledge graphs to the domain of IT gover- 
nance is novel.

The DT4GITM framework as presented in this paper is based on a synthesis of insights that we obtained from exploring the Digital Twin literature, following the first phase and partly the second phase of the methodology for developing methodological frameworks proposed in [9]: (1) identify evidence to inform the methodological framework, and (2) develop the methodological framework, (3) evaluate and refine. The first phase ensured that the framework architecture closely follows the state-of-the-art in applying the Digital Twin concept. The second phase is ongoing research, while some preliminary results are already available (e.g., [10, 11, 12]). This paper aims at providing overview and emphasizing motivation and novelty, by focusing on the framework itself, without discussing the detailed design of its individual components. To further develop and implement the framework (i.e., second and third phases), we plan to use an incremental, case-driven approach.

The remainder of the paper is structured as follows. Section 2 elaborates on the challenges of IT governance in the Digital Age. Section 3 reviews related work. Section 4 introduces the concepts of Digital Twin, Enterprise Digital Twin, and Knowledge Graph that our vision builds upon. Section 5 presents our vision on the DT4GITM framework. Section 6 illustrates, by means of a fictitious example, how an implementation of the framework (i.e., an IT governance digital twin) could operate as a flexible and responsible IT governance system. Finally, section 7 concludes the paper by stating our contribution, emphasizing the methodological and technological innovation for the domain of IT governance. It also discusses the current state of our research, its limitations, and the future research directions.

\section{Research Context}

The transition to the Digital Age brings new opportunities to organizations. However, it also introduces many potential threats and the need to adapt IT governance systems to meet new regulations and ensure compliance. For instance, the emergence of data as a key asset, in combination with the advent of advanced AI techniques, brings ample opportunities for radical, often disruptive, business model innovation. At the same time, however, it raises concerns regarding data privacy and security and more fundamental ethical concerns (e.g., explainable and ethical AI). This consequently triggers regulators to define regulatory frameworks that organizations need to comply with (e.g., GDPR for privacy and the NIS directive for security).

Furthermore, the market dynamics of the Digital Age require organizations to be more agile than ever. The in- creasing pace of change in a continuously and rapidly evolving business environment puts even more stress on IT governance because of the complexity and pervasiveness of digital transformations and the need for agility [4]. At the same time, digital transformations of organizations have a profound impact on their technological, application, data, and business infrastructures, necessitating the design of flexible IT governance systems that can adapt quickly to the new reality.

These developments exemplify only a few of the challenges that effective IT governance presently faces in the context of digital transformation. In general, developing an IT governance system requires a significant effort, which can be a barrier to adoption [4]. Take, for instance, COBIT [2, 13], which is among the most researched and implemented frameworks for IT governance [14]. COBIT provides a body of knowledge for designing and implementing not only the IT governance system, but also the IT management system that is governed. The latest version of the framework is COBIT 2019, which has been released in October 2018 as an update of the 2012 COBIT 5 version to align with the latest IT standards and compliance regulations. Although COBIT-based governance systems are organized as nested control loops requiring continuous monitoring and improvement, guided by maturity models [15], the (organisation specific) implementation process is human-driven and knowledge-intensive. Under these conditions, adoption is slow, tedious, and prone to errors [3]. Concretely, COBIT 2019 distinguishes five generic IT governance processes and 35 generic IT management processes, each with their own objectives and performance metrics, recommended practices and activities, required capability levels, roles and accountabilities, deliverables and information flows. This vast generic guidance then needs to be tailored to the specificities of individual organizations [16], aided to some extent by customization mechanisms (e.g., design factors and focus areas in COBIT 2019 [17]).

The complexity and pervasiveness of digital transformation initiatives requires a holistic view on IT governance, making it unlikely that changes are limited in scope to just one or a few processes, which may also conflict with the expected agility. Also, current governance systems are largely disconnected from the assets they control. This lack of connectedness and adaptiveness of IT governance systems is an impediment to the agile implementation of changes required for digital transformation. Therefore, it is our position that not only the primary activities of an organization should be enabled and improved by digital technologies, but that also its management and governance systems should keep pace with the digital transformation of the organization. 
This paper presents a concrete vision of how this can be done for IT governance, using the Digital Twin concept.

\section{Related Work}

The academic literature includes a number of studies that investigate how to make COBIT more contingent on factors such as the organization's size [18, 19], industry or societal sector [20, 21], and technological infrastructure [22, 23]. Making COBIT more adaptable to such contingencies does, however, not imply that the governance systems developed using COBIT also become more adaptable.

There has been some interest in introducing agility into the IT governance implementation process [24, 4, 3]. For instance, a flexible method for selecting which COBIT 2019 management or governance objectives to implement has been proposed [6]. But these studies on agile IT governance focus on new implementations of IT governance systems rather than the process of continuous adaption of already implemented systems.

A stream of research related to ours is coined smart IT governance [25], which relies on AI and Knowledge Management to leverage the rich knowledge base encapsulated in the COBIT framework. The goal of smart IT governance is to facilitate and speed up the development of IT governance systems. Although smarter design of the IT governance system does not make the system itself smart, smart IT governance provides a starting point for the vision that we elaborate on as it exploits the opportunities offered by novel digital technologies for the digitalization of IT governance. While the resulting smart advisor tool [26] takes input from the COBIT body of knowledge, we instead aim for complementing this knowledge-based approach with a data-driven approach that takes its input directly from the governed system itself, as well as from other relevant data sources.

Broadening the scope from pure IT governance or COBIT related research, the possibilities of using AI and Big Data technologies for real-time monitoring, diagnosis, and predictive maintenance of technical infrastructures was recently demonstrated [27].

Summarizing, there is a lack of research on how to adapt IT governance to address the challenges and exploit the opportunities of digital transformation. Different studies have explored aspects of this problem such as how to make IT governance implementation more agile and how to better leverage the COBIT knowledge base for designing IT governance systems, but a research gap manifests itself with respect to a comprehensive and integrated approach that combines the strengths of IT governance frameworks like COBIT (human- and knowledge-centered), data-driven technologies (real- time monitoring) and AI applications (smart and predictive governance).

\section{Background}

In this section, we provide background on the concept of Digital Twin (Section 4.1), its application for the management of organizational assets (i.e., Enterprise Digital Twin) (Section 4.2), and the concept of Knowledge Graph, which we apply for integrating and uniformly modeling the wide variety of data captured by the envisioned IT governance digital twin (Section 4.3).

\subsection{Digital Twins}

Digital Twin refers to the triad of a physical entity, a virtual entity, and the bi-directional data connections in between [7]. The virtual entity is a digital representation of the physical entity that contains all relevant properties, information, and states of the physical entity (i.e., the digital twin parameters) [28]. By leveraging computational techniques, the virtual entity allows monitoring and improving the performance of the physical entity [7]. This concept has been applied in different contexts, mostly related to manufacturing (e.g., Industry 4.0, Smart Factories) [29, 30, 27], but also other domains have been explored (e.g., Smart Cities, Smart Agriculture, Virtual Patients) [31, 32].

Two essential functions provided by digital twins are mirroring (also called twinning) and virtual processing [7]. Mirroring refers to the bi-directional connection between the virtual and physical entities and consists of two phases: metrology and realization. In the metrology phase, the state of the physical (respectively virtual) entity is observed or measured, while in the realization phase the state of the corresponding virtual (respectively physical) entity is updated. On the side of the physical entity, metrology and realization are implemented in terms of sensors (e.g., RFID tags, IoT sensors) and actuators (e.g., linear actuators, motors, relays, solenoids). An important note to make here (as relevant to our application to IT governance) is that it is recognized that also humans can play the roles of sensor and actuator [7]. Thus, the complete automation of metrology and realization is not required for mirroring, although automation leads to higher twinning rates (i.e., the speed at which the states of the virtual and physical entities are synchronized).

Virtual processing refers to the algorithms that run on the virtual entity for modeling, analyzing, optimizing, or simulating the physical entity. The effect of virtual processing on the physical entity can only be realized if the virtual entity is connected to the physical entity within a closed loop system. 


\subsection{Enterprise Digital Twins}

Early ideas on the use of digital twins in the context of organizations, resulting in Enterprise Digital Twins, have been explored in [33, 34], where the virtual entity is created from an enterprise ontology that is connected with the organization via data streams to populate it with actual data from the organization's assets. Even though the concept of Digital Twin has its roots in a desire to mirror physical entities, the potential use of the concept to mirror other phenomena, such as production processes, and indeed enterprises, has been acknowledged [7]. Recently, the application of digital twins for managing organizational assets was proposed, recognizing that these assets can be tangible (e.g., products, equipment, workers, robots) as well as intangible (e.g., services, processes) [35]. Although the notion of an intangible asset as digital twin 'physical' entity is hard to maintain, it has recently been recognized as an opportunity for digital twin applications [7]. Hence it is more appropriate to redefine digital twin physical entities as 'real-world' entities, abstracting from whether they are physical or not.

\subsection{Knowledge Graphs}

As will be clarified in the next section, the implementation of IT governance through the use of the (Enterprise) Digital Twin concept, requires the integrated and interconnected representation of different forms of digital twin parameters and other types of internal and external data, including: (1) Data about the organizational IT assets and the operational IT governance and management activities (e.g., log files); (2) Data from sources outside the organization (e.g., cloud service response times and throughput rates); (3) Explicit representations of organizational capabilities and processes (e.g., enterprise models), and data, application, and technology landscapes (e.g., enterprise-IT architecture models). These highly varied data and knowledge representations need to be integrated and uniformly modeled to create the digital twin's virtual entity.

Knowledge graphs [8, 36] have been specifically developed to enable the integration of data from heterogeneous sources. In its general form, a knowledge graph is a labeled graph, where the nodes define entities, and the edges define the relationships between those entities. The concept of Knowledge Graph, and more importantly the related computational infrastructures, were developed in the context of the Semantic Web, enabling the linking of heterogeneous data toward a unified representation that can be processed by semantic technologies and AI applications. A further benefit of knowledge graphs is that the integration is virtual, such that data redundancy and consistency issues faced by previous data integration approaches are avoided.

First ideas on transforming enterprise models into knowledge graphs, which then can act as a virtual entity in a digital twin of an organization, have recently been proposed [37]. The authors enrich ArchiMate models with domain-specific attributes that add semantics for the knowledge graph. However, neither a relationship to IT governance nor to the processing of the knowledge graphs is proposed, which differs from the DT4GITM framework as will be presented in the next section.

\section{The DT4GITM Framework}

In this section we discuss the DT4GITM framework. In Section 5.1. we start by presenting our solution objectives and solution concept. Next, in Section 5.2, we describe the design of the framework itself and elaborate on its components.

\subsection{Solution Objectives and Concept}

For IT governance to better meet the challenges of the Digital Age, it needs to become more proactive and adaptive. As discussed above, in line with our vision to digitally transform IT governance, the aim is to enable IT governance systems to be more responsive and flexible by making them smart by following a data-driven approach. These two properties are our solution objectives. More specifically, data-driven implies that the IT governance system is continuously fed by real-time data streams from the organizational IT assets, the management processes, and other potential sources such that the status, performance, and evolution of these assets can be continuously assessed. Smart means that the IT governance system is equipped with advanced analysis and prediction capabilities allowing it to act upon the organizational IT assets and management processes in both reactive and proactive modes.

Based on these objectives, we suggest to exploit the Digital Twin concept (as summarized in Section 4.1) as a basis for realizing a technological infrastructure to implement IT governance systems. The real-world entities in such IT governance digital twins are the organizational IT assets, which include tangible (e.g., a server, an application, or a database) as well as intangible (e.g., a DevOps team) assets, which need to be managed, and whose management needs to be governed. In this case, the virtual entity involves a model of (1) the organizational IT assets, (2) their management processes, and (3) the governance processes, that is kept synchronized by feeding it with data on the state and performance of these real-world entities. More importantly, the vir- 
tual entity also tracks the evolution of the IT assets and associated management and governance processes. In other words, the virtual entity not only represents a snapshot of the current situation but provides a view on its continuous evolution (like an evolving information system [38, 39]).

IT governance digital twins can be used to continuously monitor IT organizational assets and their associated management and governance processes, including the compliance to internal and external regulations. Moreover, they enable the analysis of possible risks, and the assessment of the possible impact of new or proposed regulations. Virtual processing is realized through both automated and human-triggered (i.e., interactive) model-based analyses that implement the IT governance logic. Through the mirroring function, regulating mechanisms that detect deviations from governance or management objectives or predict such deviations based on early warning signals, can act on the organizational IT assets or their management and governance processes. Furthermore, by storing historical data about the realworld entities, integrating data of external sources, and generating simulated data, the virtual processing capabilities can be further enhanced to allow for analyses and predictions that extend IT governance beyond the current guidance offered by frameworks such as COBIT.

\subsection{Framework Components}

To guide the development of IT governance digital twins, we present the DT4GITM framework (see Figure 11. This framework articulates our vision of a reference architecture for a technological infrastructure that realizes the proposed concept of IT governance digital twin. The DT4GITM framework defines the different components of such infrastructures in terms of their purpose, function, and relationships with other components or the system's environment.

We emphasize that, in this early stage of our research, the DT4GITM framework expresses our vision of applying the Digital Twin concept to IT governance. In its current shape, the proposed framework is the result of a synthesis of existing literature-based insights rather than the actual outcome of a design-based research program. The detailed design and implementation of the components, and testing and evaluation of the framework, is therefore subject to our ongoing (e.g., [10, 11, 12]) and future research.

To start with the mirroring function, the digital twin virtual entity should be able to represent (i.e., to model) the organizational IT assets and their management and governance processes. A first key question here is 'what' needs to be represented (i.e., what should be the model's contents). At the core, this question is answered by an organisation-specific ontology for IT governance, for which the DT4GITM framework, as a reference architecture, provides a domain ontology (i.e., GITM Domain Ontology in Figure 1). This domain ontology is envisioned to cover the entire governance system including (1) the system of organizational IT assets, (2) the associated management processes, and (3) the governance processes, as well as their evolution over time (within the organization). Here, the GITM Domain Ontology will follow the COBIT 2019 framework, but can be expanded to include other IT governance domain knowledge. A second key question is 'how' the relevant data and knowledge about organizational IT assets and their management and governance processes (i.e., the digital twin parameters) need to be represented. With the DT4GITM framework, we respond to this question by the GITM Knowledge Graph (see Figure 11). As discussed in the previous section, knowledge graphs have the ability to integrate and uniformly represent heterogeneous data. The GITM Knowledge Graph provides a reference how to comprehensively and uniformly represent all relevant data for IT governance including particularly data streams from COBIT Governance Processes, COBIT Management Processes, and Organizational IT Assets which can be further related to Other Data.

The basis for the virtual processing function is the twinning infrastructure that synchronizes the organization-specific ontology and the modeled organizational IT assets and their management and governance processes (i.e., virtual processing is driven by the data captured in the digital twin's knowledge graph). The operation of this function (described in the Digital Twin for GITM component, see Figure 1 follows the SenseThink-Act paradigm of Control Theory [40] and requires apart from analytical/simulation capabilities for monitoring and prediction also advanced visualization capabilities for efficient human interpretation (i.e., Workforce in Figure 1). Knowledge graph technologies provide formalization capabilities for the machine processing of the digital twin parameters and other internal and external data, to realize these virtual processing capabilities.

In the remainder of this section, we present the DT4GITM framework components in more detail.

GITM Domain Ontology - This component defines more precisely what the virtual entity will be concerned with, in terms of the concepts in the domain, their relationships and properties, as well as possible constraints. In general, within the field of Applied Ontology, a domain ontology enables one to define what (can) exist(s) in a given domain [41]. As such, the GITM Domain Ontology defines not only what the virtual entity of the IT governance digital twin will be concerned with, but 


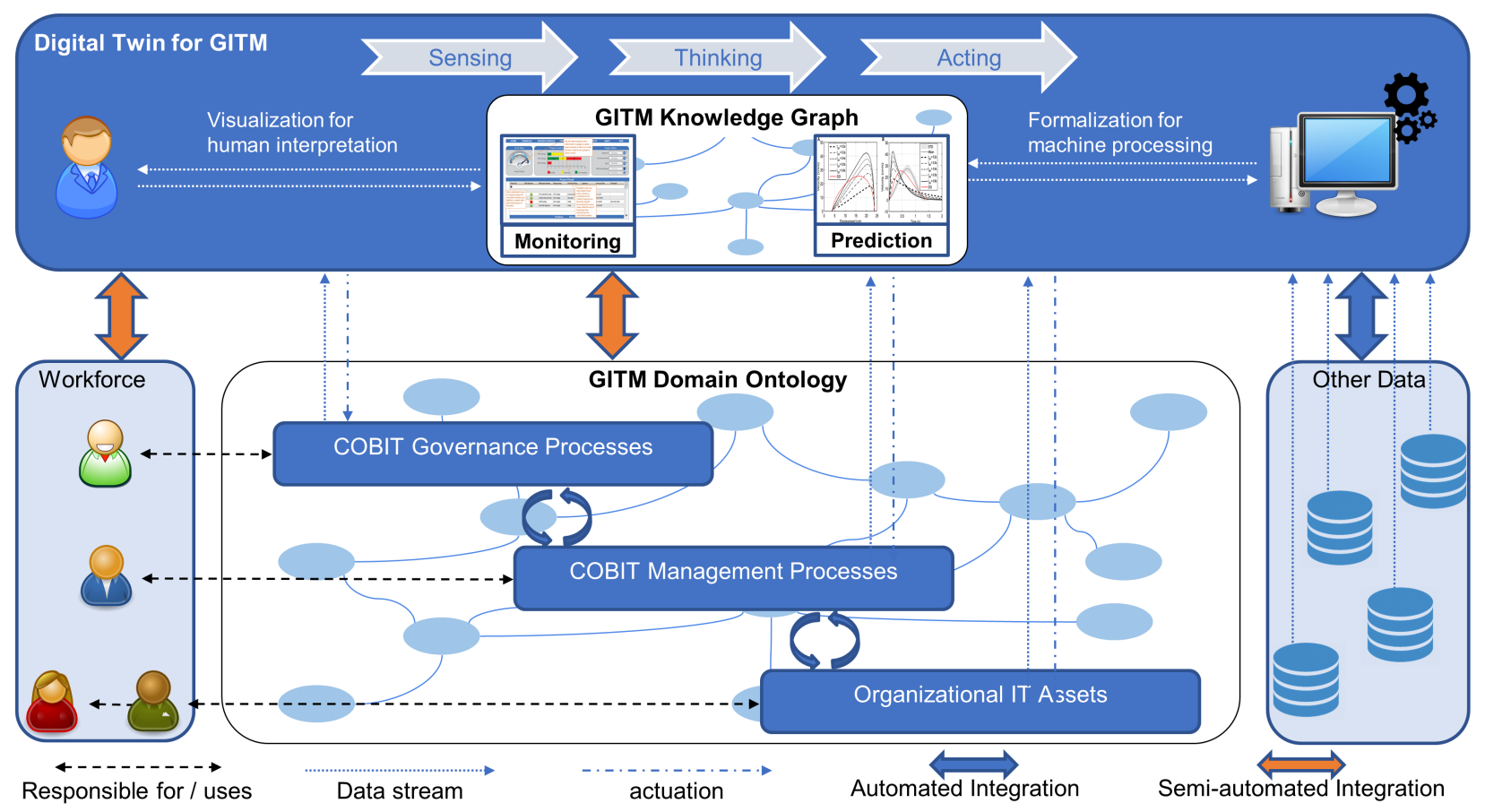

Figure 1. The Digital Twin for Governed IT Management (DT4GITM) Framework.

also the data that need to be gathered to monitor the status and performance and to track the evolution of the organizational IT assets and their management and governance processes (i.e., the IT governance digital twin parameters).

The DT4GITM framework thus offers a domain ontology as a conceptual basis for developing organization-specific ontologies for IT governance. When developing an IT governance digital twin in the context of a specific organization, the 'generic' domain ontology will need to be 'situated' to the specific concerns of that organization (e.g., privacy, security, costs). A design process that is driven by the GITM Domain Ontology, ensures that relevant IT governance concepts, and their properties, relationships and constraints, are accurately represented in the organization-specific ontology. This design process also involves defining which digital twin parameters are relevant given the specific IT governance concerns of an organization. In [12] we have presented a strategy for developing the GITM Domain Ontology as a layered ontology that is expected to become more stable as DT4GITM evolves. This will greatly help in reducing an organization's effort for designing their organization-specific ontology.

To operationalize our vision of the DT4GITM framework, as a reference architecture for developing IT governance digital twins, a GITM Domain Ontology that at least covers the concepts of COBIT 2019 is needed.
There have been some proposals for describing the ontology underlying COBIT [16, 21, 42, 43], however, to the best of our knowledge, an IT governance domain ontology that includes the concepts of COBIT 2019 has not been defined yet. Since the conceptual model underlying this framework is explicit and has been purposefully designed [17], it is feasible to develop the GITM Domain Ontology based on COBIT 2019. Although we acknowledge the challenge of ontological modeling of IT assets and their management processes, we will follow a rigorous ontology engineering methodology, building on available and relevant foundational, core and domain ontologies, and aligning our definitions with other frameworks (e.g., the ITIL Framework for IT services management [44], the TIPA Framework for process improvement of IT management processes [45]). First results in the form of concept maps of COBIT 2019 and ITIL 4 have been presented in [12].

GITM Knowledge Graph - The organization-specific IT governance ontology, for which the GITM Domain Ontology provides a design reference, provides a description of the digital twin parameters (i.e., data about organizational IT assets and their management and governance processes) that are relevant to an organization and its specific IT governance concerns. To represent these parameters and to integrate them with other relevant data and knowledge representations, including data 
from external sources (see Figure 1p, the DT4GITM framework prescribes the use of knowledge graphs.

The GITM Knowledge Graph component provides a reference of how such knowledge graphs can be constructed. Furthermore, it describes the visualization and formalization capabilities that such knowledge graphs should offer to allow for human interpretation of the information and machine processing of the data, respectively. Operationally, the GITM Knowledge Graph component consists of a set of tools that realize interactive visualizations (e.g., Visual Analytics tools, Business Intelligence dashboards) and efficient querying of the vast amounts of IT governance related data in order to support decision making.

Metrics of the COBIT framework can be operationalized as predefined queries which can be easily parameterized and executed by business users. Moreover, powerful knowledge graph platforms like Stardog 11 fed with the organization-specific IT governance digital twin knowledge graph, enable the application of advanced AI algorithms to also proactively respond to environmental or internal changes of the organizational IT assets and their management processes.

Consequently, the realization of the GITM Knowledge Graph will have to cater for the fact that the Workforce (see Figure 1) has diverse backgrounds and roles with regards to IT governance and management. The DT4GITM framework needs to make the data-driven and $\mathrm{AI}$-enabled algorithms accessible and comprehensible in adequate graphical user interfaces catering towards this diversity.

Our current research on the GITM Knowledge Graph Component will benefit from our recent exploration of transforming models into knowledge graphs [10] and performing model-based analyses with knowledge graphs [11].

Digital Twin for GITM - An organization-specific IT governance ontology details the relevant content (i.e., digital twin parameters) of the IT governance digital twin virtual entity, which is represented as a knowledge graph to allow for the integration of other data. The Digital Twin for GITM component provides guidance on how to identify the data sources to be used, how to define sensors to capture the data, how to define the data streams connecting the real-world entity with the virtual entity (i.e., how to add/update/remove data to/in/ from the knowledge graph), and how to integrate external data and other knowledge representations (e.g., enterprise (architecture) models). It also describes the processes and strategies by which decision-makers can integrate the functionalities of the IT governance digital

${ }^{1}$ https://www. stardog.com/ last visited: 03.09.2021 twin in their decision-making process.

The Digital Twin for GITM component distinguishes three types of capabilities that the technological infrastructure should realize. First, there is the mirroring function of the IT governance digital twin. Specific challenges here are the definition of sensors for different types of digital twin parameters and data sources (e.g., IoT devices for physical IT assets, autonomous software agents for systems that manage processes, queries for databases and system logs), and the integration of data of different types. Since the digital twin real-world entity is a socio-technical system, automated sensing and real-time data feeds need to be complemented with discontinuous human-based reporting and assessments. The detailed design of the component needs to address how this wide variety of inputs obtained in different ways can be integrated and implemented in the knowledge graph based virtual entity. Further, the mirroring function should be complemented by actuators to support the execution of governance and management decisions. Here also the detailed design needs to address how this can be accomplished in a socio-technical system with a primary human component (e.g., the question of which decisions can be automatically implemented and which decisions require human intervention).

A second type of capabilities are needed to realize the virtual processing function of the IT governance digital twin. The Digital Twin for GITM component describes how the IT governance system can be made smart by enhancing it with (knowledge graph based) processing and visualization capabilities. The detailed design of this component needs to address how monitoring and visual analysis capabilities can be added to the reference architecture by defining and implementing metrics that are based on the assessment mechanisms for IT governance and management objectives included in the COBIT 2019 framework.

The third type of capabilities are needed for datadriven analytics, meaning techniques that provide additional insights into the governance and management of organizational IT assets. The detailed design of the Digital Twin for GITM component should address how the rich source of data, both current and historical, captured by the IT governance digital twin virtual entity can be leveraged for purposes of organizational learning, datadriven design, and simulation of possible future worlds. It needs to be investigated how data of external sources can be integrated into the twinning infrastructure to allow for further data-driven analysis and simulation that support decision-making with respect to digital transformation initiatives. 


\section{A Scenario of an IT Governance Digital Twin in Operation}

In this section, we present a fictitious digital transformation scenario to illustrate how an IT governance digital twin that is developed using the framework, could operate as a flexible and responsive IT governance system. We emphasize that the scenario is an illustration only, as an envisioned application of the DT4GITM framework to a realistic case. For the actual evaluation of the promised benefits of the DT4GITM framework, first more research is needed to design the framework components, before an application to a real case is attempted.

A company with a longstanding tradition of delivering high-quality medical and orthopedic aids (e.g., crutches, rollators, walking sticks) throughout the European Union, is transforming itself into a provider of high-tech personal health monitoring services. Using a combination of wearables, AI algorithms, chatbots and mobile applications, users get real-time information of their health status.

Now that the company collects, for the first time, personal data about customers that use the new services, more specifically data that is highly sensitive as being related to people's health, being compliant to GDPR was identified by the Compliance Manager, as a new external compliance requirement.

After the company's Executive Committee asked the IT Governance Board to install the new role of Privacy Officer, being made responsible for GDPR compliance, one of the first actions taken by the Privacy Officer was to draft the company's data protection and privacy policy, modeled in accordance with the GDPR, and have it published on the company's website.

All new health monitoring mobile applications and also the product pages of these services on the company's website, contain links to this online policy, which specifies the rights that users of the services have in regard to their own personal data being collected by the company. The web pages detailing these rights (e.g., right of access to the data, right to erasure, right to restrict processing) are accessible to both visitors (anonymous) and registered users (after login). Furthermore, there are links to a web application that can be used for inquiries (both visitors and registered users) and to invoke rights (only registered users). The web application itself is integrated with the company's customer relationship management (CRM) system, such that responses to inquiries and follow-up interactions are stored and managed. For the moment, inquiries are responded by dedicated staff appointed by the Privacy Officer. The invocation of rights is handled by a Data Steward who collaborates with the Product Managers of the involved services. In the future, it is expected that most interactions can be handled by chatbots, based on Natural Language Processing (NLP) and Natural Language Generation (NLG) techniques, that are trained using reinforcement learning algorithms.

The IT Governance Board recognizes that the IT governance system needs to be adapted to assure that data protection inquiries and rights invocations are appropriately acted on. They hold the Compliance Manager accountable for setting targets (e.g., response times to inquiries) and monitoring their achievement. The Privacy Officer is made responsible for developing all kinds of working procedures and rules, e.g., for evaluating the clarity of the company's data protection and privacy policy.

Luckily, the company uses an IT governance digital twin developed using the DT4GITM framework. This digital twin implementation of the IT governance system can easily accommodate the required changes. It also allows to quickly observe or predict deviations from targets and respond to them.

The company's web pages, web applications, CRM system, health monitoring services, registered users of those services, user interactions, and IT-related policies are all concepts described in the organization-specific IT governance ontology or can be added to this ontology as specializations of existing concepts. New instances of these concepts (e.g., the GDPR-compliant data protection and privacy policy, inquiries) can easily be added as new nodes to the digital twin's knowledge graph. This continuous updating of the digital twin's virtual entity is established via the data stream connections of the twinning infrastructure.

The Privacy Officer uses the GITM Knowledge Graph toolset to define new metrics and visualisations, for instance, the number of visits to the GDPR pages (for visitors and registered users, per time period), the number of inquiries (for visitors and registered users, per time period, per service/app), the number of rights invocations (per type of right, per time/period, per service/ app), response times, relative frequency of escalations, etc. Deviations from targets set by the Compliance Manager can now automatically be detected (e.g., too long response times to inquiries) and appropriate actions can be taken (e.g., assigning more staff to respond to GDPRrelated inquiries). Advanced analytical tools, integrated in the IT governance digital twin, can be employed to predict deviations from objectives and trigger corrective actions. For instance, NLP techniques can be applied to GDPR-related inquiries to analyze the perceived clarity of the GDPR rights as stipulated in the policy. Sentiment analysis can be employed to assess the satisfaction with the responses. 
The example scenario illustrates how a digital twin implementation of an IT governance system might result in a flexible system as new objectives, procedures and rules for appropriately acting on GDPR-related inquiries and rights invocations can easily be integrated into the existing system. Also, the connectivity with the realworld assets and their management processes, through automated data streams with the virtual entity, increases the responsiveness of the IT governance system.

\section{Conclusion}

In this paper, we presented and illustrated a vision of how the Digital Twin concept can be used to enable IT governance to better deal with the need for more agility, flexibility, adaptivity and connectivity. We articulated our vision in terms of the DT4GITM (Digital Twin for Governed IT Management) framework. We position the DT4GITM framework as a reference architecture for developing a technological infrastructure that realizes an IT governance digital twin.

The contribution which this paper aims to make is novel in two ways. First, the application of the Digital Twin concept to developing smart and data-driven IT governance systems is novel. Second, the application of knowledge graphs and related technologies as a means to uniformly represent and integrate heterogeneous data streams in a twinning infrastructure, has not been researched so far, to the best of our knowledge. Our DT4GITM framework can be used to create a smart infrastructure for IT governance, leading to the digital transformation of IT governance itself. This concept is a genuine innovation for the IT governance domain allowing the IT governance system, the governed IT management system, and the managed system of organizational IT assets to be connected in closed loops through sensors and actuators, following the Sense-Think-Act paradigm of Control Theory [40].

As a limitation of the current state of our research, we emphasize that the DT4GITM framework expresses our vision for developing IT governance digital twins. This vision was formed based on an argumentative approach that explored the literature on Digital Twins and IT governance and combined it with the opportunities offered by the emerging technology of Knowledge Graphs. Although the conception of the DT4GITM framework is grounded in the Digital Twin literature, the framework in its current state is not yet the result of a completed research process. As a consequence, the application of the framework as it is envisioned by us, was only illustrated. Hence, the extent to which our solution achieves the objectives of delivering smart and data-driven IT governance systems, is currently lacking.
In our ongoing research [10, 11, 12], we have already advanced in further designing the conceptual backbone of the DT4GITM framework: the GITM Domain Ontology and the GITM Knowledge Graph. In our future research, we will also implement the Digital Twin for the Digital Twin for GITM component with state-of-theart technologies. We will then, following an iterative approach, define several real case-study based scenarios that will steer the design, the development, and the comprehensive evaluation of the DT4GITM framework.

\section{References}

[1] S. De Haes and W. Van Grembergen, "An Exploratory Study into IT Governance Implementations and its Impact on Business/IT Alignment," Information Systems Management, vol. 26, no. 2, pp. 123-137, 2009.

[2] S. De Haes, W. Van Grembergen, A. Joshi, and T. Huygh, Enterprise Governance of Information Technology. Achieving Alignment and Value in Digital Organizations. Springer, Heidelberg, Germany, 3rd ed., 2020.

[3] A. C. Amorim, M. Mira da Silva, R. Pereira, and M. Gonçalves, "Using agile methodologies for adopting cobit," Information Systems, vol. 101, p. 101496, 2021.

[4] S. Vejseli, D. Proba, A. Rossman, and R. Jung, "The agile strategies in IT Governance: Towards a framework of agile IT Governance in the banking industry," in Proceedings of the 26th European Conference on Information Systems, p. paper 148, AIS, 2018.

[5] M. Awais and A. Gill, "Enterprise IT Governance: Back to Basics," in Proceedings of the 25th International Conference on Information Systems Development. ISD2016, pp. 188-196, 2016.

[6] A. Fernandes, R. Almeida, and M. Mira da Silva, "A Flexible Method for COBIT 2019 Process Selection," in AMCIS 2020 Proceedings. 3, 2020.

[7] D. Jones, S. Snider, A. Nassehi, J. Yon, and B. Hicks, "Characterizing the Digital Twin: A systematic literature review," CIRP Journal of Manufacturing Science and Technology, vol. 29, pp. 36-52, 2020.

[8] D. Fensel, U. Simsek, K. Angele, E. Huaman, K. Elias, O. Panasiuk, I. Toma, J. Umbrich, and A. Wahler, Knowledge Graphs - Methodology, Tools and Selected Use Cases. Springer, Heidelberg, Germany, 2020.

[9] N. McMeekin, O. Wu, E. Germeni, and A. Briggs, "How methodological frameworks are being developed: evidence from a scoping review," BMC medical research methodology, vol. 20, no. 1, pp. 1-9, 2020.

[10] M. Smajevic and D. Bork, "Towards Graph-based Analysis of Enterprise Architecture Models," in International Conference on Conceptual Modeling, p. in press, Springer, 2021.

[11] M. Smajevic and D. Bork, "From Conceptual Models to Knowledge Graphs: A Generic Model Transformation Platform," in 2021 ACM/IEEE International Conference on Model Driven Engineering Languages and Systems Companion, p. in press, ACM/IEEE, 2021.

[12] H. A. Proper, D. Bork, and G. Poels, "Towards an Ontology-Driven Approach for Digital Twin Enabled Governed IT Management," in ODCM-DT'21: First Workshop on Ontology-Driven Conceptual Modelling of Digital Twins, Springer, 2021. 
[13] COBIT 2019 Framework: Governance and Management Objectives. Schaumberg, Illinois: ISACA, 2019.

[14] R. Debreceny and G. Gray, "IT Governance and Process Maturity: A Multinational Field Study," Journal of Information Systems, vol. 27, no. 1, pp. 157-188, 2013.

[15] D. Smits and J. v. Hillegersberg, "Diminishing the Gap Between IT Governance Maturity Theory and Practice: Renewing the Approach," Int. J. IT Bus. Alignment Gov. vol. 10, no. 1, pp. 1-21, 2019.

[16] M. Chergui, S. Adil, and M. Hicham, "IT Governance Ontology Building Process: Example of Developing $\mathrm{Au}-$ dit Ontology," International Journal of Computer Techniques, vol. 2, no. 1, pp. 134-141, 2015.

[17] D. Steuperaert, "COBIT 2019: A significant update," EDPACS - The EDP Audit, Control, and Security Newsletter, vol. 59, no. 1, pp. 14-18, 2019.

[18] J. Devos, COBIT Quickstart. IT Governance Institute, 2nd ed., 2007.

[19] T. Huygh and S. De Haes, "Exploring the Research Domain of IT Governance in the SME Context," International Journal of IT/Business Alignment and Governance, vol. 7, no. 1, pp. 20-35, 2016.

[20] L. Al Omari, P. Barnes, and G. Pitman, "Optimising COBIT 5 for IT Governance: Examples from the Public Sector," in 2nd Int. Conference on Applied and Theoretical Information Systems Research, 2012.

[21] H. Nugroho, "Conceptual model of IT governance for higher education based on COBIT 5 framework," Journal of Theoretical and Applied Information Technology, vol. 60, no. 2, pp. 216-221, 2014.

[22] M. A. Blanco and M. Piattini, "Adapting COBIT for Quantum Computing Governance," Communication in Computer and Information Science, no. 1266, pp. 274283,2020

[23] D. Henriques, R. Pereira, I. S. Bianchi, R. Almeida, and M. Mira da Silva, "How IT Governance can assist IoT project implementation," International Journal of Information Systems and Project Management, vol. 8, no. 3, pp. $25-45,2020$

[24] A. C. Amorim, M. M. da Silva, R. Pereira, and M. Gonçalves, "Using SCRUM for Implementing IT Governance with COBIT 5," in Proceedings of the 22nd International Enterprise Distributed Object Computing Conference. IEEE, pp. 198-207, 2018.

[25] M. Chergui and A. Chakir, "IT Governance Knowledge: From Repositories to Artificial Intelligence Solutions,' Journal of Engineering Science and Technology Review, vol. 13, no. 5, pp. 67-76, 2020.

[26] M. Chergui and A. Chakir, "IT GRC Smart Advisor: Process Driven Architecture Applying an Integrated Framework," Advances in Science, Technology and Engineering Systems Journal, vol. 5, no. 6, pp. 247-255, 2020.

[27] B. Palchevskyi and L. Krestyanpol, "The Use of the "Digital Twin" Concept for Proactive Diagnosis of Technological Packaging Systems," in Data Stream Mining \& Processing (S. Babichev, D. Peleshko, and O. Vynokurova, eds.), pp. 432-444, Springer, 2020.

[28] D. Adamenko, S. Kunnen, R. Pluhnau, A. Loibl, and A. Nagarajah, "Review and comparison of the methods of designing the Digital Twin," in Proceedings of the 30th CIRP Design, pp. 27-32, 2020.
[29] K. Y. H. Lim, P. Zheng, and C.-H. Chen, "A state-of-theart survey of Digital Twin: techniques, engineering product lifecycle management and business innovation perspectives," Journal of Intelligent Manufacturing, vol. 31, pp. 1313-1337., 2020.

[30] T. Y. Melesse, V. Di Pasquale, and S. Riemma, "Digital Twin Models in Industrial Operations: A Systematic Literature Review," Procedia Manufacturing, vol. 42, pp. 267-272, 2020.

[31] Dassault Systèmes, "Meet Virtual Singapore, the city's 3D digital twin," 2018.

[32] K. Bruynseels, F. Santoni de Sio, and J. van den Hoven, "Digital Twins in Health Care: Ethical Implications of an Emerging Engineering Paradigm," Frontiers in Genetics, vol. 9, no. article 31, 2018.

[33] S. Barat, V. Kulkarni, T. Clark, and B. Barn, "An actor based simulation model for analyzing complex business systems," in Proceedings of the IEEE 2019 Winter Simulation Conference, pp. 157-168, 2019.

[34] V. Kulkarni, S. Barat, and T. Clark, "Towards adaptive enterprises using digital twins," in Proceedings of the IEEE 2019 Winter Simulation Conference, pp. 60-74, 2019.

[35] M. Dietz and G. Pernul, "Digital Twin: Empowering Enterprises Towards a System-of-Systems Approach," Business \& Information Systems Engineering, vol. 62, no. 2, pp. 179-184, 2020.

[36] M. Galkin, S. Auer, and S. Scerri, "Enterprise Knowledge Graphs: A Backbone of Linked Enterprise Data," in 2016 IEEE/WIC/ACM International Conference on Web Intelligence (WI), pp. 497-502, 2016.

[37] M. Kubelskiy, "Application of Semantic Networks and Enterprise Architecture Approaches for Creation of Digital Twins of Organizations," in Networks in the Global World V (A. Antonyuk and N. Basov, eds.), (Cham), pp. 144-161, Springer International Publishing, 2021.

[38] M. Jarke, J. Mylopoulos, J. W. Schmidt, and Y. Vassiliou, "DAIDA: An Environment for Evolving Information Systems," ACM Transactions on Information Systems, vol. 20, pp. 1-50, January 1992.

[39] H. A. Proper and T. P. van der Weide, "A General Theory for Evolving Application Models," IEEE Transactions on Knowledge and Data Engineering, vol. 7, pp. 984996, December 1995.

[40] M. Siegel, "The sense-think-act paradigm revisited," in Robotic Sensing, 2003. ROSE'03. 1st International Workshop on, pp. 5-10, June 2003.

[41] G. Guizzardi, Ontological Foundations for Structural Conceptual Models. PhD thesis, University of Twente, Enschede, the Netherlands, 2005.

[42] M. Goeken and S. Alter, "Representing IT Governance Frameworks as Metamodels," in CSREA EEE, pp. 4854, 2008.

[43] L. Moudoubah, A. El Yamami, K. Mansouri, and M. Qbadou, "Towards the implementation of an ontology based on COBIT Framework (CobitOntology)," in Proceedings of the 1st International Conference on Smart Systems and Data Science, pp. 1-6, 2019.

[44] Axelos, ITIL Foundation Handbook. The Stationery Office, London, UK, 2015.

[45] B. Barafort, ITSM Process Assessment Supporting ITIL (TIPA). Van Haren Publishing, Zaltbommel, the Netherlands, 2009. 